\title{
Self-similarity in Touch with Stochastic Process
}

\author{
Krzysztof Z. Sokalski \\ Institute of Computer Science, Cz, estochowa University of \\ Technology, Al. Armii Krajowej 17, 42-200 Cz, estochowa, Poland \\ Email: ksokalski76@gmail.com
}

Partial differential representation of self-similarity feature has been derived from notion of the homogenous function in general sense. This representation allows consideration of stochastic selfsimilar systems. As well as the partial differential representation allows consideration of Stochastic Partial Differential Equation describing stochastic Self-similar system.

Keywords: scaling; self-similarity; stochastic processes

\section{INTRODUCTION}

Representation of the self-similarity by homogenous function in general sense [1] leads to general forms of solutions for problems obeying scaling. Moreover, analytic form of this function is constrained only by the definition of homogeneity in general sense. Therefore, there are many ways to propose mathematical model possessing enough free parameters for fitting of model's coefficients which make agreement between mathematical model and experimental data [2]. However, this approach does not cover other important problems. For instance, does the power losses remain to be self-similar in presence of stochastic process?. To answer this question we need stochastic partial differential equation for the power losses depending on frequency, pick of magnetic induction and function model of stochastic process. For purpose of this paper we select for further considerations the problem of power density losses in Soft Magnetic Materials [2].

\section{APPROACH TO SCALING IN SOFT MAGNETIC MATERIALS (SMM)}

Let $P_{\text {tot }}\left(f, B_{m}\right)$ be a function describing the power losses density, where $f$ and $B_{m}$ are independent variables describing frequency and pick of magnetic induction, respectively. Applying the scaling hypothesis to $P_{t o t}\left(f, B_{m}\right)$, we derive the most general deterministic form for the power losses,

$$
B_{m}^{-\beta} P_{t o t}\left(f, B_{m}\right)=F\left(B_{m}^{-\alpha} f\right) .
$$

$P\left(f, B_{m}\right)$ satisfying $(1)$ is called the uniform function in general sense. Magnitudes obeying scaling are self-similar[1]. There are many examples of applications of the self-similarity, for example [1], [2], [3] and others.

\section{SCALING REPRESENTED BY THE PARTIAL DIFFERENTIAL EQUATION}

Let us calculate partial derivatives of $P_{t o t}$ with respect to $B_{m}$ and $f$ :

$$
\frac{\partial P_{t o t}}{\partial f}=B_{m}^{\beta-\alpha} F^{\prime}\left(\frac{f}{B_{m}^{\alpha}}\right),
$$

where

$$
F^{\prime}\left(\frac{f}{B_{m}^{\alpha}}\right)=B_{m}^{\alpha-\beta} \frac{\partial P_{t o t}}{\partial f}
$$




$$
F\left(\frac{f}{B_{m}^{\alpha}}\right)=B_{m}^{-\beta} P_{t o t}
$$

Using the set of the following relations $\{(2),(3),(4)\}$ and eliminating the uknown function $F(\cdot)$ as well as its derivative, we derive the following linear, partial, differential equation $(\mathrm{PDE})$ of the first order:

$$
B_{m} \frac{\partial P_{t o t}}{\partial B_{m}}=\beta P_{t o t}-\alpha f \frac{\partial P_{t o t}}{\partial f} .
$$

Solving this equation we get the solution of the following form:

$$
P_{t o t}\left(f, B_{m}\right)=B_{m}^{\beta}\left(\frac{f}{B_{m}^{\alpha}}\right)^{z},
$$

where $z$ is an arbitrary real number not equal zero. (5) is linear equation, therefore any linear combination of (6) solutions for different values of $z$ satisfies (5). On the other hand, PDE representation of scaling is equivalent to the representation by the homogenous function in general sense if $F\left(\frac{f}{B_{m}^{\alpha}}\right)$ in (4) is an analytic function. The presented achievement will enable us to formulate the stochastic partial differential equation for self-similar stochastic system. It is necesseary to point out that (5) is similar to equation representing self-similarity in relativistic quantum field theory [5].

\section{INFLUENCE OF STOCHASTIC PROCESSESS ON SELF-SIMILAR SYSTEM}

Let us consider the self-similar system characterized by the following stochastic partial differential equation:

$$
\hat{L} P\left(f, B_{m}\right)=a \frac{\partial^{2} W}{\partial \xi \partial \tau}
$$

where

$$
\hat{L}=B_{m} \frac{\partial}{\partial B_{m}}-\beta+\alpha f \frac{\partial}{\partial f}
$$

is operator corresponding to $(5)$ and $W_{f, B_{m}}$ denotes the additive stochastic term. Induction and fequency variables are subjected to the following domains:

$$
f_{0} \leq f \leq f_{1}, \quad B_{m, 0} \leq B_{m} \leq B_{m, 1}
$$

Boundary for $P\left(f, B_{m}\right)$ were formulated in forms of the Cauchy problems, as the two independent alternatives:

$$
P\left(f_{0}, B_{m}\right)=u_{0}\left(B_{m}\right), \text { or } P\left(f, B_{m, 0}\right)=g_{0}(f) .
$$

Applying the Green function method we write down formal solution of (7) [4].

$$
P\left(f, B_{m}\right)=P_{0}\left(f, B_{m}\right)+a \int_{f_{0}}^{f} d \xi \int_{B_{m, 0}}^{B_{m}} d \tau G\left(f, B_{m} ; \xi, \tau\right) W_{\xi, \tau}
$$

Where homogeneous solution satisfies $\hat{L} P_{0}\left(f, B_{m}\right)=0$ and definition of the Green function reads:

$$
\hat{L}^{-1} \delta(\xi-f) \delta\left(\tau-B_{m}\right)=G\left(f, B_{m} ; \xi, \tau\right)
$$


The stochastic term in the two dimensions is of the following form:

$$
W_{\xi, \tau}=\frac{\partial^{2} W}{\partial \xi \partial \tau}
$$

The frequency $f$ or $\xi$ and the pick of magnetic induction $B_{m}$ or $\tau$ variables are independent and (13) can be factorized:

$$
W_{\xi, \tau}=W_{\xi} W_{\tau}
$$

where

$$
W_{\xi}=\frac{d C_{\xi}}{d \xi} \quad W_{\tau}=\frac{d C_{\tau}}{d \tau}
$$

and $C_{\xi}$ is the Brownian movement for the $\xi$ coordinate. To determine the statistical moments of the solution (11) we use :

$$
<W_{\xi} W_{\tau}>=<W_{\xi}><W_{\tau}>=0 .
$$

Inserting $(14,15)$ to $(11)$ we derive:

$$
P\left(f, B_{m}\right)=P_{0}\left(f, B_{m}\right)+a \int_{f_{0}}^{f} d C_{\xi} \int_{B_{m}, 0}^{B_{m}} G\left(f, B_{m} ; \xi, \tau\right) d C_{\tau} .
$$

Performing average of (17) with respect to $d C_{\xi}$ and $d C_{\tau}$ we derive mean value of the solution:

Applying standard methods [4] we derive the first moment of $P\left(f, B_{m}\right)$ :

$$
<P\left(f, B_{m}\right)>=P_{0}\left(f, B_{m}\right) .
$$

To complete set of the basic statistics we calculate the variance of the solution (17),

$\operatorname{Var}\left(P\left(f, B_{m}\right)\right)=a^{2}\left\langle\int_{f 0}^{f} d C_{\xi} \int_{f 0}^{f} d C_{\zeta} \int_{B m 0}^{B m} d C_{\tau} \int_{B m 0}^{B m} d C_{\theta} G\left(f, B_{m} ; \xi, \tau\right) G\left(f, B_{m} ; \zeta, \theta\right)\right\rangle$

Assuming the Dirac delta expansion for average in (19)[4]:

$$
<d C_{\xi} d C_{\zeta} d C_{\tau} d C_{\theta}>=<d C_{\xi} d C_{\tau}><d C_{\zeta} d C_{\theta}>=d \xi d \theta \delta(\xi-\zeta) \delta(\tau-\theta)
$$

we derive the following formula for the variance:

$$
\operatorname{Var}\left(P\left(f, B_{m}\right)\right)=a^{2} \int_{f 0}^{f} d \xi \int_{B m 0}^{B m} d \tau G^{2}\left(f, B_{m} ; \xi, \tau\right) .
$$

\section{CONCLUSION}

Note that $P_{0}\left(f, B_{m}\right)$ is the self-similar process. Therefore, the average of $P\left(f, B_{m}\right)$ inherits the self-similarity from the solution of homogeneous equation,

$$
\hat{L} P_{0}\left(f, B_{m}\right)=0 .
$$

The second moment reveals that the variance exists only for square integrable Green functions, which is general criterion for existence of stochastic solutions for Stochastic 
Partial Differential Equations. However, main topic of the presented here project is to investigate influences of stochastic processes on power losses in magnetic materials. Full analysis of correlations between the self-similar processes and different stochastic processes is in progress.

[1] G.I. Barenblatt, Scaling, Cambridge Texts in Applied Mathematics, Cambridge University Press 2003.

[2] K.Z. Sokalski, B. Ślusarek, and J.Szczygłowski, Scaling in Magnetic Materials, Chapter in Magnetic Materials, Editor: K.Maaz, Intech Rijeka 2016.

[3] K.Z. Sokalski, J. Szczygłowski, M. Najgebauer and W. Wilczy nski, Losses scaling in soft magnetic materials, COMPEL: Int. J. Comput. Math. Electr. Electron. Eng.,26,640-649( 2007).

[4] D. Henderson, P. Plaschko, Stochastic Differental Equations in Sciences and Technology, World Scientific 2006, New Jersey.

[5] D.V. Shirkov, The Bogolubov Renormalization Group, arXiv:hep-th/9602024v1 6Feb 1996.

(C) 2017 by the author; licensee Preprints, Basel, Switzerland. This article is an open access article distributed under the terms and conditions of the Creative Commons by Attribution (CC-BY) license (http://creativecommons.org/licenses/by/4.0/). 